
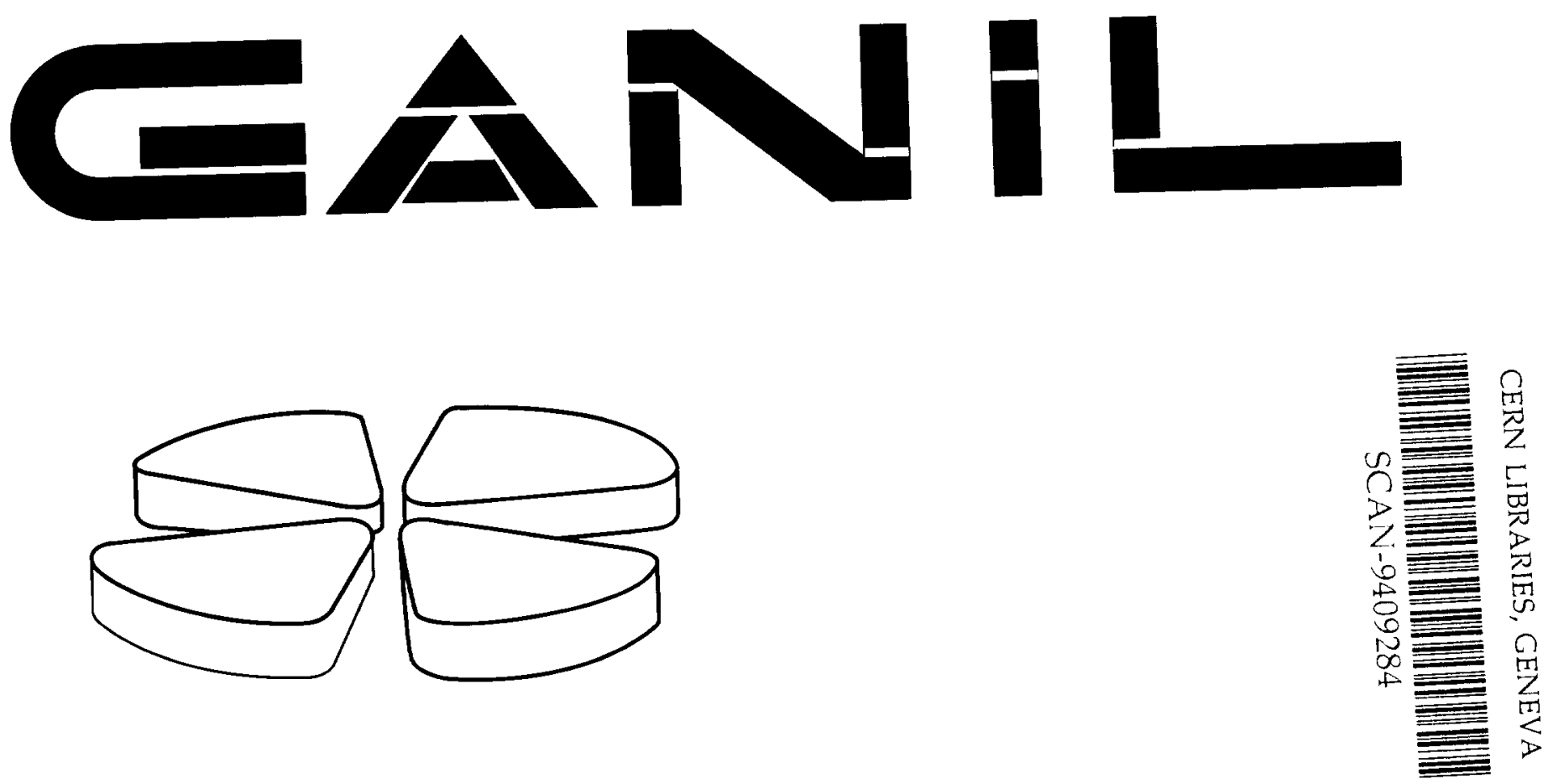

Invited talk at the Fifth International Conference on Nucleus-Nucleus Collisions, Taormina, Italy, 30 May-4 June 1994.

Sw $w+0$

Study of the nuclear multifragmentation : recent results obtained with the INDRA detector in the intermediate energy domain.+

F. SAINT-LAURENT for the INDRA Collaboration*

GANIL, BP 5027, 14021 Caen, FRANCE 
. 
Invited talk at the Fifth International Conference on Nucleus-Nucleus Collisions, Taormina, Italy, 30 May-4 June 1994.

\title{
Study of the nuclear multifragmentation : recent results obtained with the INDRA detector in the intermediate energy domain.+
}

\author{
F. SAINT-LAURENT for the INDRA Collaboration* \\ GANIL, BP 5027, 14021 Caen, FRANCE
}

The new $4 \pi$ multidetector INDRA, designed for the study of hot nuclear systems decaying by multifragmentation, is available for experiments since the beginning of 1993 . First results emphasize its very high detection capabilities. Preliminary results on multiplicity distributions and elemental charge distributions in the most violent collisions for the ${ }^{36} \mathrm{Ar}+58 \mathrm{Ni}$ and ${ }^{129} \mathrm{Xe}+$ nat $\mathrm{Sn}$ systems are presented.

\section{INTRODUCTION}

The disassembly of highly excited nuclear systems is one of the most challenging topics of heavy-ion dynamics. The multifragmentation process, corresponding to the simultaneous emission of several intermediate mass fragments (IMF), has received a great deal of both theoretical and experimental attention during the last few years. Such a process is expected to occur in the deexcitation of very hot nuclear systems formed in heavy ions collisions at intermediate energy.

Following one of the models, the hot system, formed during the initial stage of the reaction, expands under the action of thermal pressure and/or decompression cycle following the initial compression. Upon entering a region of sufficiently low density $\left(\rho=0.2-0.3 \rho_{0}\right)$, mechanical instabilities, connected to the existence of a liquid-gas phase transition for bulk nuclear matter, may develop and cause a prompt disassembly of the system into many

\footnotetext{
* B. Berthier, J.L. Charvet, R. Dayras, E. De Filippo, R. Legrain, L. Nalpas, E. Pollacco, C. Volant, CEA-DAPNIA, CE Saclay, 91191 Gif S/Yvette, France.

G. Auger, A. Benkirane, J. Benlliure, A. Chbihi, P. Ecomard, A. Le Fèvre, N. Marie, J. Pouthas, F. Saint-Laurent, J.P. Wieleczko, GANIL, BP5027, 14021 Caen, France.

C.O. Bacri, B. Borderie, P. Box, L. Lakehal-Ayat, A. Ouatizerga, E. Plagnol, M.F. Rivet, M. Squalli, L. Tassan-Got, IPN Orsay, IN2P3-CNRS, 91406 Orsay Cedex, France. R. Bougault, R. Brou, J. Colin, D. Cussol, D. Durand, A. Genoux-Lubain, C. Le Brun, J.F. Lecolley, O. Lopez, M. Louvel, M. Mahi, V. Métivier, J. Péter, R. Regimbart, E. Rosato, J.C. Steckmeyer, B. Tamain, E. Vient, A. Wielock, K. Yuasa-Nakagawa, LPC Caen, ISMRA et Université, 14050 Caen Cedex, France.

P. Eudes, D. Gourio, J.L. Laville, A. Rahmani, T. Reposeur, LPN Nantes, IN2P3-CNRS et Université, 44072 Nantes Cedex, France.

A. Demeyer, D. Guinet, P. Lautesse, L. Lebreton,

IPN Lyon, IN2P3-CNRS et Université, 69622 Villeurbanne Cedex, France

+ Experiment performed at GANIL.
} 
fragments [1]. Such a process would be expected to occur at bombarding energies of $\approx 40$ $60 \mathrm{MeV} / \mathrm{u}$ for symmetric systems and on a time scale of the order of $100-200 \mathrm{fm} / \mathrm{c}$ [2]. These dynamic instabilities may equally result from density fluctuations [3], not directly related to a phase transition.

Another possibility for the system is to evolve into strongly deformed and exotic shapes thus favouring multifragment decomposition [4]. For very heavy systems, Coulomb repulsive forces may also induce instabilities which are also predicted to result in multifragment emission [5]. Last but not least, statistical models of equilibrated nucleus decay predict an increase in the fragment emission probability with increasing excitation energy. This fragment emission is described either by a standard sequential binary statistical decay [6], or by a simultaneous break-up of the system $[7,8,9]$, the latter strongly dependent on the "freeze out" volume.

In front of the numerous possible scenarii, careful experimental and theoretical investigations are clearly needed. A strong experimental effort has been devoted to the characterization of this disintegration process. These studies require an event by event detection capability of high precision and efficiency. Many of the methods and models used in the analysis of the data also imply a proper determination of the size of the excited system as well as a precise measurement of the size (charge, mass) and energy of the decay products (e.g. ref.[10]). The $4 \pi$ multidetector INDRA was built to study in details these aspects of the multifragmentation processes $[11,12]$. Very efficient detection techniques - Ionization chamber, silicon and scintillator detectors - were associated to satisfy the main goals of the detector :

- high geometrical efficiency $(\approx 90 \%)$,

- identification threshold down to very low energy $(\approx 1 \mathrm{MeV} / \mathrm{u})$,

- identification of $\mathrm{p}, \mathrm{d}, \mathrm{t},{ }^{3} \mathrm{He}$ and ${ }^{4} \mathrm{He}$ up to $\approx 200 \mathrm{MeV} / \mathrm{u}$,

- charge identification up to $\mathrm{Z}=50$.

In this paper, we will report on some very recent results obtained with the INDRA detector. Because the analysis (identification and energy calibration) of the data has not yet been completed, the data presented here are preliminary.

\section{EXPERIMENTAL RESULTS}

\subsection{Multiplicity distributions.}

The ${ }^{36} \mathrm{Ar}+\mathrm{KCl},{ }^{36} \mathrm{Ar}+{ }^{58} \mathrm{Ni},{ }^{129} \mathrm{Xe}+{ }^{\text {nat }} \mathrm{Sn}$ and ${ }^{155} \mathrm{Gd}+{ }^{238} \mathrm{U}$ systems were studied over the $30-95 \mathrm{MeV} / \mathrm{u}$ energy range. A complete $\mathrm{Z}$ identification was achieved for element ranging from 4 to 64 , and isotopic separation was carried out for $Z=1$ to 4 .

The simplest variable which can be extracted from the data is the charged product multiplicity $N_{c}$, including light charged particles $(Z=1,2)$ as well as heavier fragments. This variable is a good measure of the violence of the collision. The larger the charged product multiplicity, the more violent the collision is. For all the studied systems, more violent collision occur at higher bombarding energy as no saturation of the charged product multiplicity is observed $[13,17]$.

Contour plots showing the correlation between the number of IMF's (defined as $\mathrm{Z} \geq 3$ ) and $N_{C}$ are displayed in figure 1 for several systems. The general trend is an increase of the number of IMF's with of the total number of charged products. More and more fragments (light particles and IMF) are produced when the collision becomes more and more violent. However, various behaviours are observed, depending on the system size. For the light ${ }^{36} \mathrm{Ar}+58 \mathrm{Ni}$ system, although the charged product multiplicity increases with the bombarding energy, the IMF multiplicity does not evolve from 40 to $95 \mathrm{MeV} / \mathrm{u}$. As already observed for the 
similar $\mathrm{Zn}+\mathrm{Ti}$ system [14], this experimental fact could be associated to a reduction of the mean fragment size when increasing the violence of the collision, and might be an indication that the vaporization stage (break-up into $\mathrm{H}$ and $\mathrm{He}$ isotopes only) is reached for this system. At $95 \mathrm{MeV} / \mathrm{u}$, up to 35 charged products are collected, to be compared with the available charge $\mathrm{Z}_{\max }=46$.

For the heavy ${ }^{129} \mathrm{Xe}+{ }^{\text {nat }} \mathrm{Sn}$ system, the IMF multiplicity as well as the charged fragment multiplicity strongly increase from 25 to $50 \mathrm{MeV} / \mathrm{u}$, as already reported by many other studies [15]. The trends exhibited in the heaviest ${ }^{155} \mathrm{Gd}+238 \mathrm{U}$ system are similar to that observed for the $50 \mathrm{MeV} / \mathrm{u}{ }^{129} \mathrm{Xe}+{ }^{\text {nat }} \mathrm{Sn}$ reaction. For multiplicity lower than 10 , a contamination due to the carbon backing of the target is present. This contamination will be easily removed by the requirement of a complete event detection.

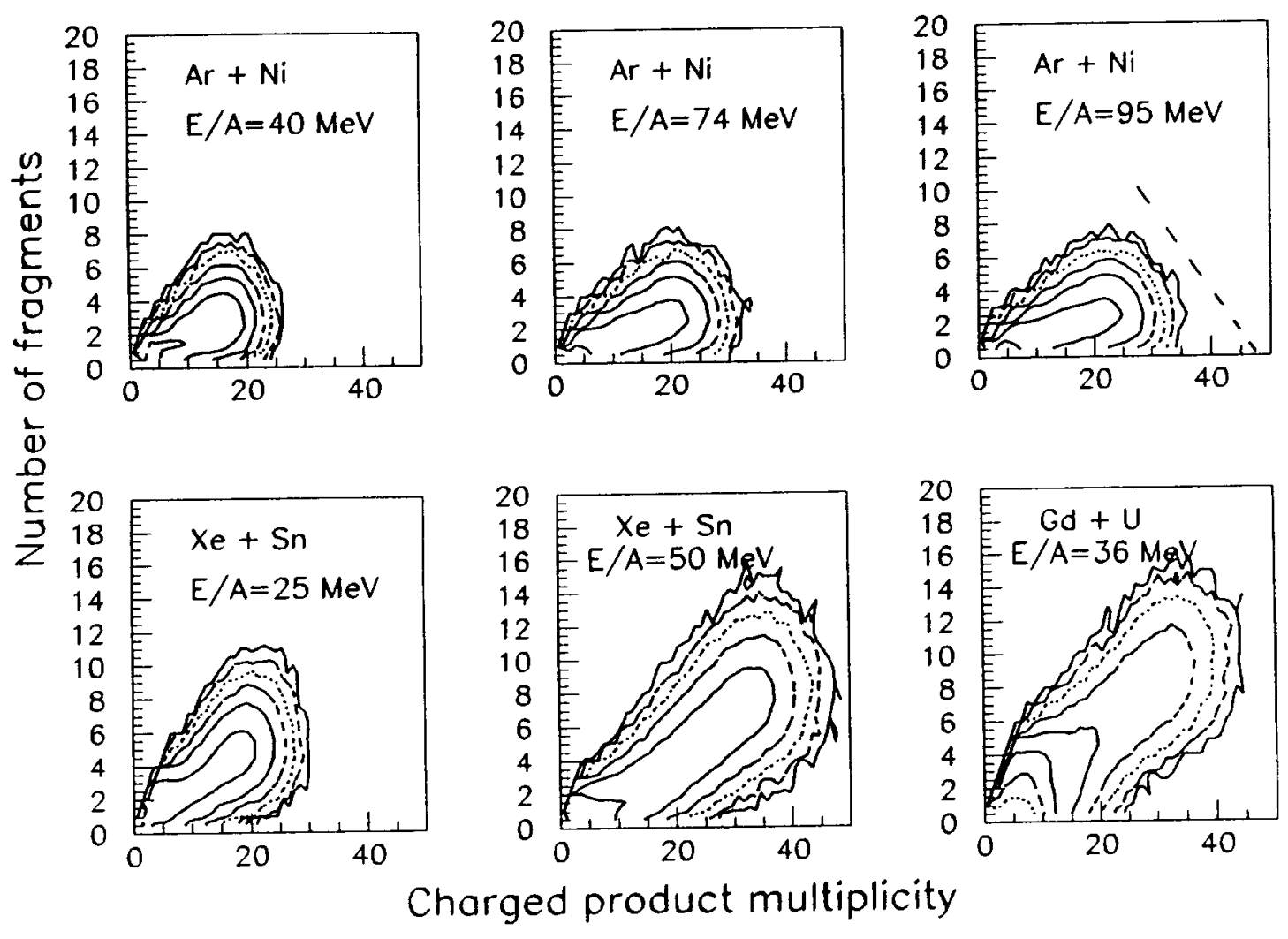

Figure 1 : Contours plots showing the relationship between the total charged product multiplicity and the IMF multiplicity $(Z \geq 3)$ for several systems. The dashed line at $95 \mathrm{MeV} / \mathrm{u}$ $36 \mathrm{Ar}+58 \mathrm{Ni}$ reaction indicates the $Z$ conservation assuming $Z=3$ for all fragments and $Z=1$ for all light charged particles

Predictions of the QMD model [16], after filtering by detection efficiency, reproduce very well the ${ }^{36} \mathrm{Ar}+{ }^{58} \mathrm{Ni}$ data at low energy as well as at $95 \mathrm{MeV} / \mathrm{u}$ [17]. On the other hand, the strong increase of the number of IMF's when going from 25 to $50 \mathrm{MeV} / \mathrm{u}$ for ${ }^{129} \mathrm{Xe}+{ }^{\text {nat }} \mathrm{Sn}$ system is not properly reproduced by the calculations. Only an almost constant number of IMF's is predicted. This may be a hint for a too small energy transfer during the collision : not enough excitation energy is imparted to the fragments, leading to a lower rate of IMF's emission as compared to the data. The subsequent decay of primary emitted fragments may also increase the final number of IMF's [18]. 


\subsection{Charge distributions.}

Figure 2 displays the contour plots showing the charge $\mathrm{Z}_{\max }$ of the heaviest fragment detected in one event as a function of $\mathrm{N}_{\mathrm{C}}$ for the ${ }^{129} \mathrm{Xe}+$ natSn system at 25 and $50 \mathrm{MeV} / \mathrm{u}$. The left hand panels are minimum bias data and the right hand panels correspond to the requirement that more than $80 \%$ of the total charge are detected. About 10 to $15 \%$ of total recorded events remain after such a selection, depending on the energy. This value demonstrates the very high detection efficiency of INDRA. From unbias data (left hand part), one observes two branches at small multiplicity : the one starting at $Z \approx 54$ corresponds to peripheral collisions where the quasi-projectile remnant is detected ; the branch at small $Z_{\max }$ is associated with badly measured events where neither the projectile-like nor the target-like nuclei are detected. These two branches merge at large multiplicity. For complete events, only large multiplicity events are kept.
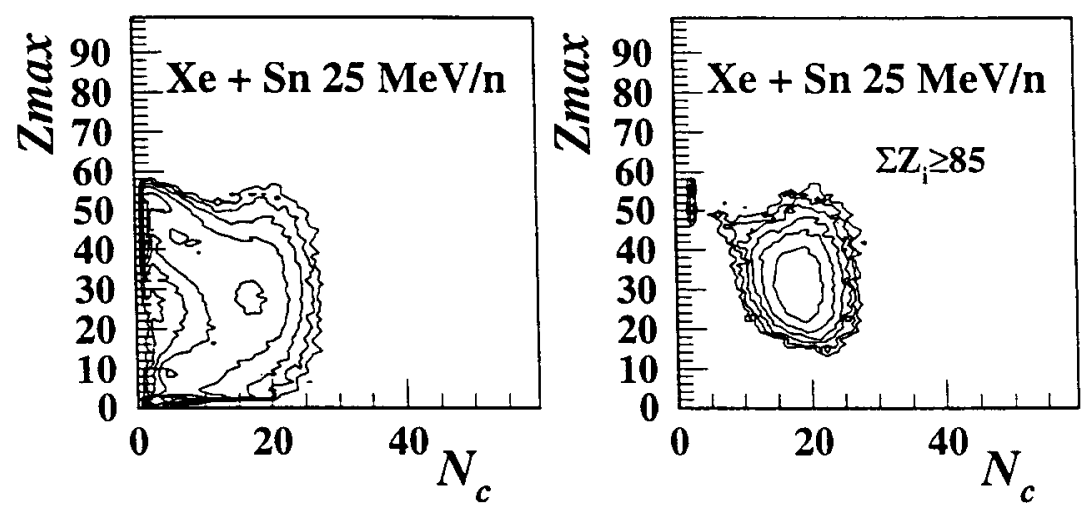

Figure 2: Contours plots showing the relationship between the total charged product multiplicity and the charge of the heaviest fragment in ${ }^{129} \mathrm{Xe}+$ natSn at 25 and $50 \mathrm{MeV} / \mathrm{u}$. Left hand part is from
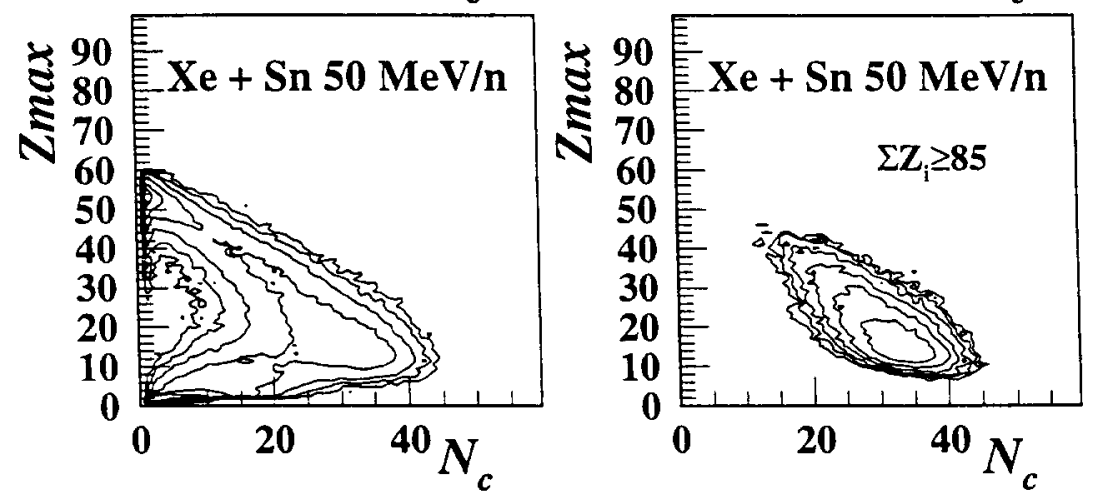
minimum bias data. Right hand part corresponds to events having more than $80 \%$ of the total charge detected. Preliminary data.

The properties of the $\mathrm{Z}$ distribution of a well defined class of events can put constraints on the models. For well measured events (right hand part), the multiplicity dependence of $Z_{\max }$ strongly evolves when increasing the incident energy. At $25 \mathrm{MeV} / \mathrm{u}$, a heavy residue is still present. Its average charge is $\left\langle\mathrm{Z}_{\max }\right\rangle \approx 33$, weakly depending on the multiplicity. At $50 \mathrm{MeV} / \mathrm{u}$, a strong multiplicity dependence is observed : $\left\langle\mathrm{Z}_{\max }\right\rangle$ decreases from 40 to 14 as $\mathrm{N}_{\mathrm{c}}$ increases from 15 to 40 . For the more violent collisions associated to the highest multiplicities, the mean number of IMF is 8 (see fig. 1). For these events, the three heaviest fragments exhaust about $1 / 3$ of the available total charge and have roughly the same size. The remaining charge is equally shared by the light charged particles and the 5 lighter IMF's.

The $\mathrm{Z}_{\max } v s \mathrm{~N}_{\mathrm{c}}$ correlations for the ${ }^{36} \mathrm{Ar}+{ }^{58} \mathrm{Ni}$ reaction at 32 and $95 \mathrm{MeV} / \mathrm{u}$ are displayed in the top of figure 3 . We observe the same qualitative evolution with the bombarding energy as 
for the ${ }^{129} \mathrm{Xe}$ on nat $\mathrm{Sn}$ induced reactions. However, at $95 \mathrm{MeV} / \mathrm{u}$, the average $Z_{\max }$ value is smaller $\left(<Z_{\max }>\approx 4\right)$, indicating that only fragments of very small size are produced.
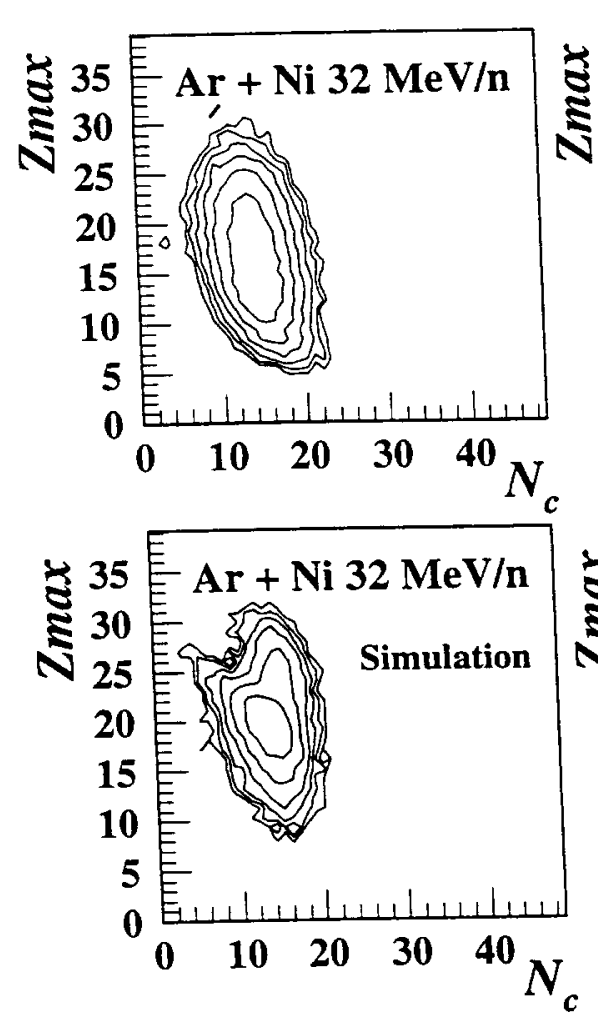
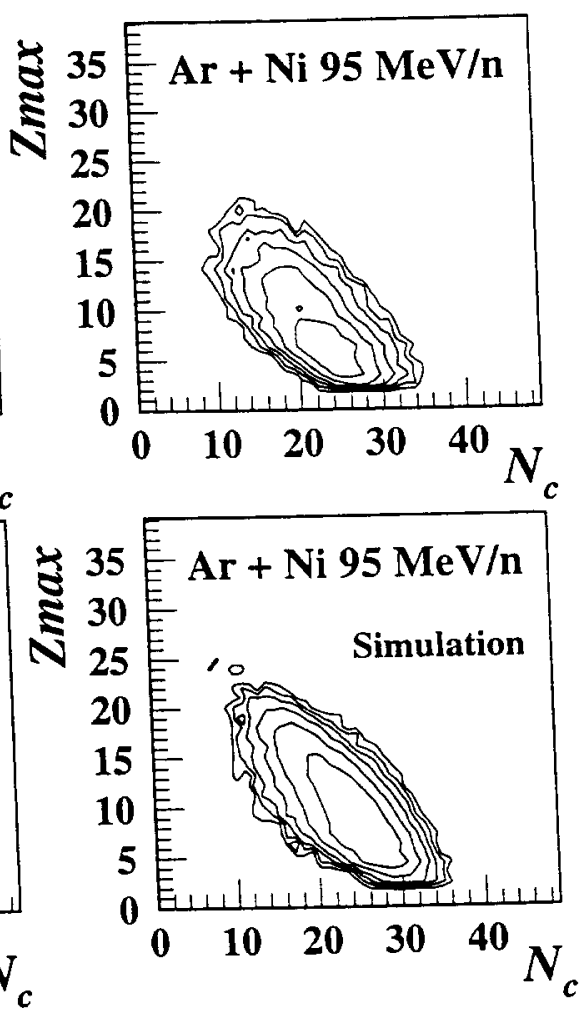

Figure 3 : Contours plots showing the relationship between the total charged product multiplicity and the charge of the heaviest fragment in ${ }^{36} \mathrm{Ar}+58 \mathrm{Ni}$ at 32 and $95 \mathrm{MeV} / \mathrm{u}$. Data for a near complete events detection are shown in the upper part. Results from the GENEVE simulator [19] are displayed in the bottom part. Preliminary data.

Results of calculations using the event generator GENEVE [19] are presented in the bottom of figure 3 . In this calculation, the formation and the deexcitation of the hot nuclei produced in the reaction are decoupled. The overlap of the projectile and the target in coordinate and momentum spaces determines the rate of preequilibrium emission for each impact parameter. Then, the dissipative phase is described assuming a binary process for impact parameters greater than the value for which the pocket in the interacting potential dissappears. For lower impact parameters, a fusion-like process is assumed. In case of binary collisions, the amount of dissipated energy and angular momentum is estimated using a parametrization of results obtained in the framework of kinetic equations as BUU or LV. Finally, the deexcitation of the hot nuclei is described by using a statistical approach in which a sequential binary decay including all channels from neutron emission to symmetric fragmentation is assumed.

Such a simple model reproduces reasonably well the correlation between $\mathrm{N}_{c}$ and the IMF multiplicity for the ${ }^{36} \mathrm{Ar}+{ }^{58} \mathrm{Ni}$ system over the whole studied energy range (not shown). Furthermore, the energy and multiplicity dependence of the heaviest fragment distribution are also well reproduced (fig 3 ).

The probability distributions of the detected charged products for ${ }^{36} \mathrm{Ar}+{ }^{58} \mathrm{Ni}$ at several bombarding energies are presented in figure 4 . In each panel, the results obtained for different charged product multiplicity gates are displayed. At $32 \mathrm{MeV} / \mathrm{u}$, whatever the multiplicity is, one observes a steep falloff in yield between $Z=1$ and $Z=4$, followed by a constant yield over a large $Z$ range. This range decreases however when the total multiplicity increases, due to 
charge conservation. These results agree with elemental charge distribution for the similar ${ }^{40} \mathrm{Ca}+{ }^{40} \mathrm{Ca}$ system at $35 \mathrm{MeV} / \mathrm{u}[20]$.

At higher bombarding energies, this constant yield for fragments is only observed for low multiplicity gates, and over a rather smaller $Z$ range. For larger multiplicities, a steeper and steeper decrease of the fragment yield is observed when increasing the multiplicity. These results might be a first indication of a change in the reaction mechanism for the more violent collisions between 32 and $52 \mathrm{MeV} / \mathrm{u}$.

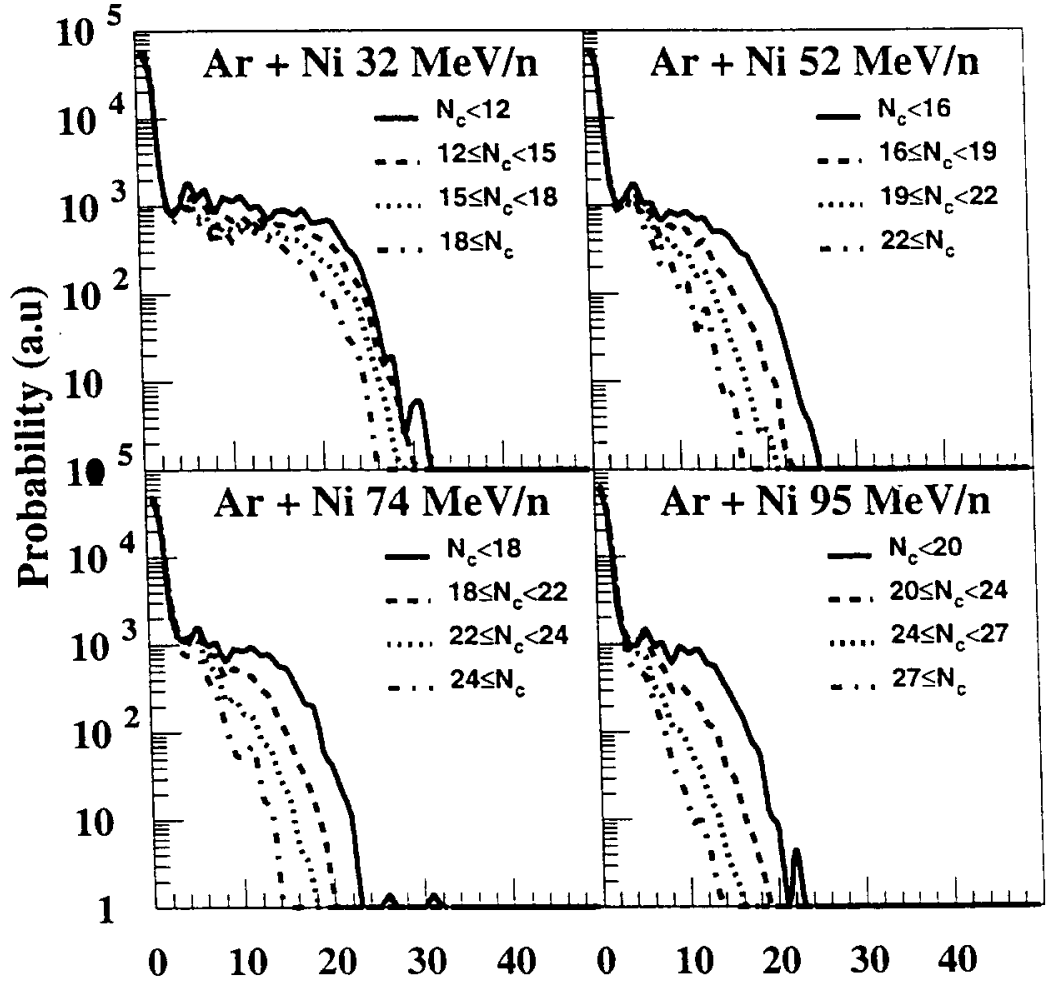

Figure 4:C harge probability distribution for the 32, 52,74 and $95 \mathrm{MeV} / \mathrm{u} 36 \mathrm{Ar}+58 \mathrm{Ni}$ reactions. Distributions for different total multiplicity gates as indicated in the figure are displayed. Preliminary data.

\section{Charge}

\subsection{Dalitz plots.}

To investigate the size sharing of three-body decays (i.e. events in which exactly 3 IMFs are detected), we use the Dalitz representation [21] : each event is represented by a point in a triangle, in a way that the distances to the sides of the triangle correspond to the values $Z_{1} / Z_{t o t}$, $\mathrm{Z}_{2} / \mathrm{Z}_{\text {tot }}, \mathrm{Z}_{3} / \mathrm{Z}_{\mathrm{tot}}$, where $\mathrm{Z}_{\text {tot }}$ is the sum of $\mathrm{Z}_{1}, \mathrm{Z}_{2}$ and $\mathrm{Z}_{3}$. In such a plot, an event with one large fragment and two small ones is located in one of the corners, while an event with two mediumsize fragments and a small one is located in the middle of one side of the triangle. Finally the center of the triangle is populated when the three fragments have about the same size.

The Dalitz plots obtained for ${ }^{36} \mathrm{Ar}+{ }^{58} \mathrm{Ni}$ at 32,52 and $95 \mathrm{MeV} / \mathrm{u}$ are shown in the figure 5. Complete events containing only three $\mathrm{Z} \geq 3$ fragments are required with a further selection on the total charged product multiplicity. At each incident energy, the multiplicity threshold was adjusted to keep $10 \%$ of the total reaction cross-section. For the more violent collisions at $32 \mathrm{MeV} / \mathrm{u}$, only the corners of the triangle are populated (fig. 5a). This indicates that at low excitation energy, the excited nuclear system, undergoing three fragments emission, 
strongly prefers to decay into one heavy and two light fragments. The pattern at $52 \mathrm{MeV} / \mathrm{u}$ is completely different (fig. $5 \mathrm{~b}$ ) : for the same selected class of events only the center of the Dalitz plot is populated. This pattern does not evolve anymore when increasing the bombarding energy up to $95 \mathrm{MeV} / \mathrm{u}$ (fig. 5c). When selecting less violent collisions, a low excitation energy pattern is recovered (fig. 5d). Beside this pattern evolution, the sum of the 3 fragment charges $Z_{\text {tot }}$ strongly decreases from 22 to 12 between 32 and $52 \mathrm{MeV} / \mathrm{u}$, and evidently follows a softer evolution up to $95 \mathrm{MeV} / \mathrm{u}$, as by definition $\mathrm{Z}_{\mathrm{tot}} \geq 9$.

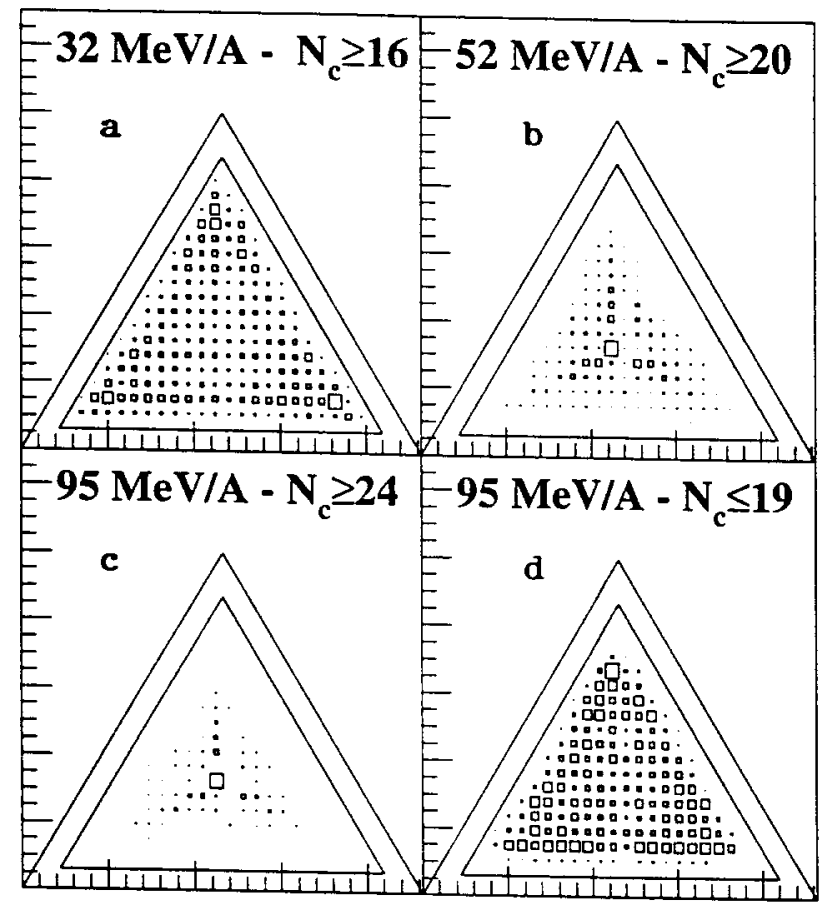

Figure 5 : Dalitz plots for events in which exactly 3 IMFs are detected for the 32, 52 and 95 $\mathrm{MeV} / \mathrm{u}{ }^{36} \mathrm{Ar}+58 \mathrm{Ni}$ reaction. More violent collisions are selected for panels $a, b$ and $c$. A less violent collision selection is also shown at $95 \mathrm{MeV} / \mathrm{u}(\mathrm{d})$. Preliminary data.

The findings extracted from the Dalitz plots and from the elemental charge distributions seem to indicate a transition between 32 and $50 \mathrm{MeV} / \mathrm{u}$ in the disintegration mechanism for central collisions in the ${ }^{36} \mathrm{Ar}+58 \mathrm{Ni}$ reaction.

In the framework of the Landau-Vlasov equation, dynamical calculations were performed for the neighbouring ${ }^{40} \mathrm{Ca}+{ }^{40} \mathrm{Ca}$ system between 40 and $100 \mathrm{MeV} / \mathrm{u}$ for central collisions [2]. These simulations predicted that, after a compression phase, the system undergoes an expansion phase and reaches low density region $\left(\rho / \rho_{0}<0.4\right)$. Above $40-50 \mathrm{MeV} / \mathrm{u}$, the system enters the spinodal region of mechanical instabilities. At $50 \mathrm{MeV} / \mathrm{u}$, instabilities develop and a dynamical fragmentation of the system is predicted. It is remarkable that the incident energy' predicted for this transition corresponds to the one experimentally found for the evolution of central collision mechanism.

The clusterization from a low density nuclear matter region has been qualitatively studied by $\mathrm{Ph}$. Chomaz et al [22]. If the system enters the spinodal region, they found that, due to the finite nuclear force range, the fluctuations which develop cannot have short wave length. Consequently small size fragment formation is inhibited and a production of nearly equal size fragments is expected. These qualitative predictions have to be quantified but are again consistent with the observed trends in the Dalitz plots. 


\subsection{Search for vaporization events.}

We can take advantage of the very high detection efficiency of INDRA to search for rare events. Events containing only light particles might be good canditates to study the vaporization of the very hot system formed. In one of the proposed scenarii, the rapid expansion under thermal pressure may lead the system to a very low density region where the fragment formation is inhibited [9]. In this case, a dissociation of the system into its elemental constituants is expected. We have isolated nearly complete events $\left(\mathrm{Z}_{10 \mathrm{t}} \geq 80 \%\right.$ of the total charge) containing only Hydrogen and Helium isotopes. These events have necessarly a high multiplicity, and thus originate from very violent collisions. At $32 \mathrm{MeV} / \mathrm{u}$, not such events are found. When the bombarding energy exceeds $52 \mathrm{MeV} / \mathrm{u}$, the probability of finding such events increases rapidly to reach about $12 \%$ of the most violent collisions class at $95 \mathrm{MeV} / \mathrm{u}$.

Between 52 and $95 \mathrm{MeV} / \mathrm{u}$, the experimental mean multiplicity of Helium, $\mathrm{N}_{\mathrm{He}}$, decreases from 13.5 to 11 , while the Hydrogen one, $N_{H}$, increases from 10 to 16 . The corresponding mean $\mathrm{N}_{\mathrm{He}} / \mathrm{N}_{\mathrm{H}}$ ratio evolves from 1.35 near the threshold production, to 0.7 at $95 \mathrm{MeV} / \mathrm{u}$. This ratio has been calculated including all light particles, whatever their production mechanism is. For example, a strong preequilibrium component may affect the $\mathrm{N}_{\mathrm{He}} / \mathrm{N}_{\mathrm{H}}$ ratio at $95 \mathrm{MeV}$. Nevertheless, the very large number of He isotopes found at $52 \mathrm{MeV} / \mathrm{u}$ is surprising. A possible explanation might be found considering the $Q$-values of various decay channels. The decay of a heavy residue remnant of $Z \approx 8-12$ into numerous alpha particles is energetically favoured. Further kinematical analysis will be performed in order to verify whether such $\mathrm{N}_{\mathrm{He}} / \mathrm{N}_{\mathrm{H}}$ ratio evolution gives more information on the afterburning stage of the multifragmentation process.

\section{CONCLUSION}

Begun in 1990, INDRA has successfully acted in march-april 1993. The $36 \mathrm{Ar}+\mathrm{KCl}$, ${ }^{36} \mathrm{Ar}+{ }^{58} \mathrm{Ni},{ }^{129} \mathrm{Xe}+$ nat $\mathrm{Sn}$ and ${ }^{155} \mathrm{Gd}+{ }^{238} \mathrm{U}$ systems have been studied over the $30-95 \mathrm{MeV} / \mathrm{u}$ energy range. we have presented new results obtained for the ${ }^{129} \mathrm{Xe}+$ nat $\mathrm{Sn}$ and ${ }^{36} \mathrm{Ar}+{ }^{58} \mathrm{Ni}$ systems. The very high detection capabilities of the apparatus offers the possibility to select a large number of nearly complete events. A further selection of events containing a large number of charged products was made in order to isolate a class of more violent collisions.

For ${ }^{129} \mathrm{Xe}+$ nat $\mathrm{Sn}$ central collision events, the mean charge of the heaviest fragment decreases from 34 to 15 when increasing the incident energy from 25 to $50 \mathrm{MeV} / \mathrm{u}$. The associated average number of IMF is 6 and 8 respectively. Further investigations will be made in order to study the charge (or size) division in multifragmentation events.

For ${ }^{36} \mathrm{Ar}+{ }^{58} \mathrm{Ni}$ central collisions, the charge distribution and the Dalitz plot exhibit a dramatic change between 32 and $52 \mathrm{MeV} / \mathrm{u}$, indicating a possible evolution of the reaction mechanism in this energy range. At the lower energy, the data can be understood in the framework of a statistical decay of an incompletely fused system leading to a residual nucleus. At $52 \mathrm{MeV}$, no residual nucleus survives the collision. This finding cannot be reproduced by a conventional statistical binary decay model including a preequilibrium phase. Whatever the energy, the model predicts a heavy residue associated to two light fragments. These results might be an experimental evidence of a dynamical effect as predicted by Landau-Vlasov simulations. Nevertheless, deeper experimental investigations are clearly needed to confirm these findings.

For ${ }^{36} \mathrm{Ar}+{ }^{58} \mathrm{Ni}$, complete events containing only Hydrogen and Helium isotopes were isolated. These events are possible candidates for a vaporization of the system. 
Acknowledgement : This work is a collective work. None of the results presented here would have been available without the strenuous effort of each member of the INDRA collaboration. Furthermore, special thank to R. Roy and Z Majka for fruitful discussions during the preparation of this talk.

\section{REFERENCES}

1. J. Aichelin and H. Stöcker, Phys. Lett. B176 (1986) 14

2. E. Suraud, M. Pi, P. Schuck, B. Remaud, F. Sébille, C. Grégoire, F. Saint-Laurent, Phys. Lett. B229 (1989) 359.

3. D. Boal and J.N. Glosli, Phys. Rev. C37 (1988) 91; Phys. Rev. C42 (1990) R502

4. L.G. Moretto et al, Phys. Rev. Lett. 69 (1992) 1884

5. B. Borderie, B. Remaud, M.F. Rivet and F. Sébille, Phys. Lett. B302 (1993) 15

6. R.J. Charity et al, Nucl. Phys. A483 (1988) 371

7. J. Bondorf, R. Donangelo, I.N. Mishustin and H. Schulz, Nucl. Phys. A444 (1985) 460

8. D.H.E. Gross, Rep. Prog. Phys. 53 (1990) 605 and references therein.

9. W.A. Friedmann, Phys. Rev C42 (1990) 667 W.A. Friedmann, Advances in Nucl. Dynamic 1993, ed. by W. Bauer and B. Back.

10. X. Campi, Phys. Lett. B208 (1988) 351

11. E. Plagnol and the INDRA Collaboration, Nouvelles de Ganil n 44 (February 1993) J. Pouthas, in preparation.

12. B. Borderie and the INDRA Collaboration, XXXIth Meeting on Nuclear Physics, Bormio (Italy), January 1993.

F. Saint-Laurent and the INDRA Collaboration, GANIL P93-11, International SchoolSeminar on Heavy-Ion Physics, Dubna (Russia), May 1993.

13. F. Saint-Laurent and the INDRA Collaboration, XXII Hirschegg workshop, H. Feldmeier \& W. Nörenberg Ed.(1994)

J. Benlliure et al, Nouvelles de Ganil nº 49 (1994) 3

14. A. Kerambrun, Thesis, Caen 1993, unpublished.

15. D.R. Bowman et al, Phys. Rev. C46 (1992) 1834

16. J. Aichelin, Phys. Rep. 202 (1991) 233

17. J.C. Steckmeyer et al, Cont. to the XXXIInd Meeting on Nuclear Physics, Bormio (Italy), January 1994.

18. G. Pielert et al, Phys. Rev C46 (1992) 1457

J. B. Natowitz, XXII Hirschegg workshop, H. Feldmeier \& W. Nörenberg Ed.(1994)

19. J.P. Wieleczko, E. Plagnol and P. Ecomard, Cont. to the II TAPS Workshop, Guardamar June 93, ed. by J. Diaz and Y. Schutz, World Scientific, and to be published.

20. K. Hagel et al, Phys. Rev. Lett. 68 (1992) 2141

21. G. Bizard et al, Phys. Lett. B302 (1993) 162

22. Ph. Chomaz, contribution to this conference. 
